Sharif University of Technology
Scientia Iranica
SCIENTIA
IRAN Transactions B: Mechanical Engineering
www.scientiairanica.com

\title{
A computational model for estimation of mechanical parameters in chemotactic endothelial cells
}

\author{
A. Kiyoumarsioskouei, A. Shamloo*, S. Azimi, M. Abeddoust and M.S. Saidi \\ School of Mechanical Engineering, Sharif University of Technology, Tehran, Iran.
}

Received 9 September 2014; received in revised form 29 December 2014; accepted 25 April 2015

\section{KEYWORDS}

Cell motility;

Chemotaxis;

Reaction-diffusion

model;

Cell cytoeskeleton

simulation.

\begin{abstract}
A cell migration numerical simulation is presented to mimic the motility of endothelial cells subjected to the concentration gradients of a Forebrain embryoniccortical neuron Conditioned Medium (CM). This factor was previously shown to induce the directional chemotaxis of endothelial cells with an over-expressed G protein coupled receptor 124 (GPR 124). A cell simulator program incorporates basic elements of the cell cytoskeleton, including membrane, nucleus and cytoskeleton. The developed 2D cell model is capable of responding to concentration gradients of biochemical factors by changing the cytoskeleton arrangement. Random walk force, cell drag force and cell inertial effects are also implemented into the cell migration to complete the simulation of the phenomenon. The obtained results of cell migration were calibrated with experimental cell chemotaxis data. This model can be implemented for prediction of cell behavior during cell chemotaxis and also provides a powerful tool to explain the cell migration phenomenon, mechanistically.

(C) 2016 Sharif University of Technology. All rights reserved.
\end{abstract}

\section{Introduction}

Cell migration plays a pivotal role in numerous biological phenomena such as wound healing, embryogenesis, cancer invasion and inflammatory response. It is a highly complex process in which different elements of the cell, including actin filaments, surface signaling receptors and microtubules, are involved. Though highly investigated by different researchers in biology and engineering sciences, the mechanisms underlying the migration of cells are still unidentified in many aspects. Finding the answers to some fundamental questions in this area would, undoubtedly, have a great impact on different fields of biology.

Different types of cell demonstrate diverse behavior during migration and thus, proposing a single unique mechanism that can be generalized for all types

\footnotetext{
*. Corresponding author. Tel.: +98 2166165691 ;

Fax: +982166165599

E-mail address: shamloo@sharif.edu (A. Shamloo)
}

of cell is impossible in practice. However, the cells are similar in exhibiting cyclic intracellular processes while migrating [1]. After being stimulated by a migration promoting agent, such as the concentration gradient of a biochemical factor, at the first step, the migrating cell polarizes and extends some protrusions in the direction of the imposed gradient. Actin filament polymerization creates lamellipodia and filopodia that become stabilized by adhering to the extracellular matrix (ECM) or the neighboring cell surfaces [2]. At the second step, the lamellipodia and filopodia at the rear part of the cell attenuate their adhesion to the ECM. Finally, the polarized cell moves forward. The cells repeat this cycle until the external signal diminishes and they receive no stimuli to polarize.

Among the signals which stimulate directional migration of the cells, the gradient of the biochemical species in the external media of the cell is of high importance. The chemotaxis of neutrophils is the underlying factor in the body's immune reaction. They migrate to the location of infection or injury because 
of a chemical signal they receive from other cells in the immune system or the microbes at the infection site [3]. As a matter of fact, almost all cells sense extracellular biochemical signals, and many of them react by migrating in response [4].

Various mathematical models have been proposed to simulate the cell migration process and also various computational models have been developed based on these mathematical models. Meinhardt proposed one of the basic and primary computational models for cell chemotaxis simulation [5]. He attributed the cells directional sensitivity to an inherent system inside the cells that translates the extracellular signals of the gradients of chemo attractants into the formation of an intracellular pattern, which, in turn, results in the extension of lamellipodia and filopodia towards the source of biochemical factors. Similar to the model of Gieier and Meinhardt, many researchers, studying the chemotaxis in eukaryotic cells, considered a hypothetical "compass needle" mechanism inside the cells that governs the formation of new pseudopods towards the chemo attractant [6-9]. While these compass based models are able to represent some aspects of cell chemotaxis successfully, many observations show that they are usually limited to steep gradients of the chemo attractant since, in shallow gradients, the cell are more controlled by their internal dynamics rather than chemo attractants. In order to overcome this shortcoming, Neilson et al. proposed a pseudopodbased computational model that simply attributes cell chemotaxis to the movement of pseudopods on the cell surface, not a compass inside the cells [10]. In their model, the pseudopods themselves respond to the chemo attractant molecules through a positive or negative feedback loop, and, so, orient cell migration.

Also, Andrew and Insall have shown that many chemotactic cells form a protrusion that splits in a Y-like manner into two branches, only one of which eventually survives [11]. Their study and related works have demonstrated very complex behavior during cell movements and have claimed that it is not possible to consider all the details of cell chemotaxis in a mathematical model $[12,13]$. Therefore, in this work, the first target is to simulate cell chemotaxis using a simple mathematical model in such a way that this phenomenon is consistent with experimental data.

In the present study, the numerical simulation of cell motility during the chemotaxis of endothelial cells is performed. The constant biochemical factor concentration gradient around the cell stimulates the cell simulator. This simulator is a simple model for a cell which includes nucleus, cytoskeleton, and membrane. Intracellular mechanics explains how the cell simulator is developed, and, finally, the results are discussed.

\section{Interacellular mechanics}

In order to investigate the effects of an imposed constant concentration gradient, a model is required to simulate cell polarization and migration. The study of cell chemotaxis might be possible by considering every cell as a single point. However, simulation of the cytoskeletal components would not only give a better physical representation of the phenomenon, but the details of the migrating cell's cytoskeleton would also be appreciable, and, thus, helpful for future studies like cell proliferation and group migration phenomena. Kiyoumarsioskouei et al. have offered a 3-dimensional endothelial cell model to describe a numerical method for simulating the cell cytoskeleton [14]. In the current study, three basic elements of a cell; elastic plasma membrane, cell cytoskeleton, and cell nucleus, are considered in the model. The cell membrane and nucleus are initially divided into $N_{0}$ elements, each of which is modeled by a damper and a spring arranged in parallel (Voigt Model). The damper accounts for the viscous effects, while the spring is responsible for mimicking the elastic behavior of cell elements. This model for investigating cellular and subcellular behavior was previously utilized by Jamali et al. [15] and showed strong capabilities in representation of subcellular behavior, including cell motility. Each node on the cell is connected to the corresponding point on the cell nucleus using a Voigt unit, as depicted in Figure 1. In the same manner, each node on the cell membrane and cell nucleus are connected to their two neighboring nodes.

Acting forces on each element are presented in Eq. (1):

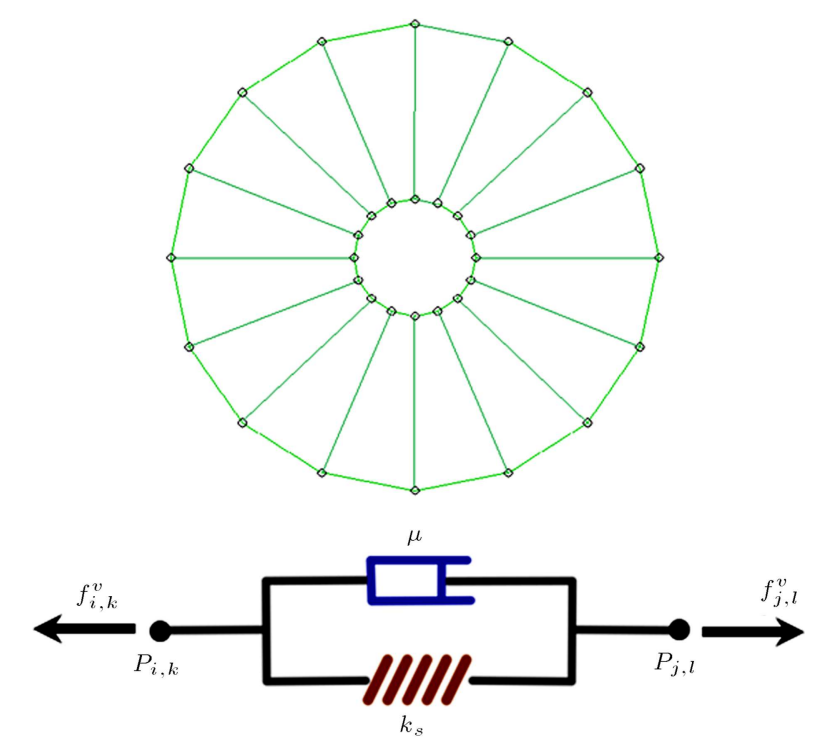

Figure 1. Basic discretized elements of a cell (up) by Voigt units (down). Each element of the discretized cell that connects two nodes consists of a spring and a damper in parallel. 


$$
F_{i, k}^{\mathrm{tot}}=F_{i, k}^{\mathrm{inner}}+F_{i, k}^{\mathrm{cell}-\mathrm{cell}}+F_{i, k}^{\mathrm{cell}-\mathrm{ECM}}+F_{(i, k)}^{\mathrm{ext}} .
$$

$F_{i, k}^{\text {tot }}$ is the total force acting on a node, $F_{i, k}^{\text {inner }}$ is the total force resulting from the inner elements of the cell, $F_{i, k}^{\text {cell-cell }}$ is the force due to a neighboring cell, $F_{i, k}^{\text {cell-ECM }}$ is the force exerted on the node by the ECM, and $F_{i, k}^{\text {ext }}$ is the result of an external force like a magnetic field. All mentioned forces will be discussed in detail in the following sections.

\subsection{Inner force calculation}

The total force acting on a node on the membrane resulting from interaction with other nodes of the inner parts is the sum of three forces: The membrane Voigt units' forces, the cytoskeleton forces and the force due to the inside pressure of the cell. The forces due to the membrane and cytoskeleton are the sum of spring and damper forces. The spring force, Eq. (2), is proportional to changes in its length, and the proportionality factor was adopted from [15]:

$$
F_{\text {spring }}=k\left(\sqrt{\left(X_{j+1}-X_{j}\right)^{2}+\left(Y_{j+1}-Y_{j}\right)^{2}}-l_{0}\right)
$$

where $l_{0}$ is the initial length of the Voigt springs, and $X$ and $Y$ are the coordinates of nodes connected by the Voigt member.

The damper force is proportional to the inner product of velocity and position vectors, as shown schematically in Figure 2.

$$
F=\mu \overrightarrow{\mathbf{V}} \cdot \hat{\mathbf{r}},
$$

where $\overrightarrow{\mathbf{V}}$ is the velocity vector and $\hat{\mathbf{r}}$ is the normalized position vector.

Moreover the membrane is subjected to a force that is a result of the pressure difference between the inside and outside of the cell. The magnitude of this force is:

$$
F_{i, j}^{m, n}=\left(P_{\text {in }}-P_{\text {out }}\right) R / 2,
$$

and its direction is normal to the cell surface.

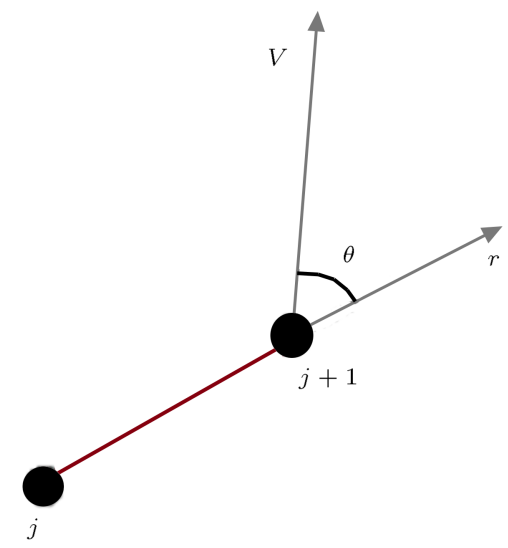

Figure 2. In calculating viscous forces, only the component of velocity in the direction of Voigt nodes contributes to viscous forces.

\subsection{Components of cell motility}

Cell shape and position change continuously as a result of different cell processes, like cell growth, cell migration, mitosis, apoptosis etc.

During cell migration, the cell is polarized and specific protrusions form around its body. The migration of the cell in this study is the result of the concentration gradient of biochemical factors. The important question that arises here is how do the cells polarize, make protrusions and finally migrate in the direction of the imposed concentration gradient.

It is agreed by many researchers that cell migration during chemotaxis is the outcome of four components, Eq. (5) [1]. The first three components account for cell activities as a living organism. The first component that contributes to cell migration is the effect of biochemical factors. Another component is cell memory while migrating. In other words, cells are willing to continue movement in their former direction of migrating while subjected to a gradient in a new direction. The third component is the random motion cells demonstrate while migrating. Finally, the last component is the drag force that affects every object in a fluid flow. In other words, the first three components will disappear if we hypothetically assume that the cells are nonliving objects, while the last component is always present, regardless of cells being assumed living or nonliving.

$$
\begin{aligned}
& \vec{V}_{m}=\theta \vec{V}_{c}+(1-\theta) \vec{V}_{m}+\vec{V}_{r}+\vec{V}_{d}, \\
& \theta \text { : Blending factor. }
\end{aligned}
$$

When the gradient of a biochemical factor is imposed on the cells, the density of related receptors for that factor in the membrane is amplified in the leading edge [16]. As an example, Narang et al. utilized the reaction-diffusion model, established by Gierer and Meinhardt, to simulate the accumulation of $\mathrm{PI}(3,4,5) \mathrm{P}_{3}[6]$. However, the set of partial differential equations they have established is stiff. They have proposed four equations, which all are nonlinear and highly coupled. As a result, their model is highly expensive in terms of computational cost. For the current study, a simple linear model is employed in which only the gradient direction is considered. For the sake of simplicity, the gradient magnitude is assumed to be constant through the extracellular medium. This assumption is reasonable for the current study, as the gradient of biochemical species is constant. Therefore, the $\vec{V}_{c}$ component of velocity can be generated by the following scheme:

1. The gradient direction is sensed by the cell simulator;

2. The location of the leading edge due to the external signal is defined; 


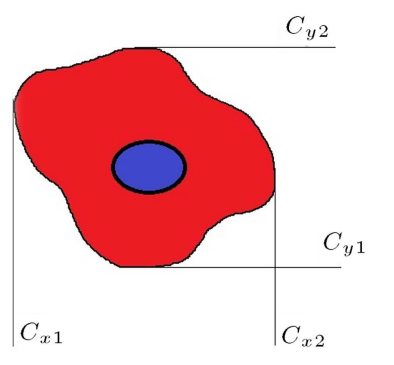

(a)

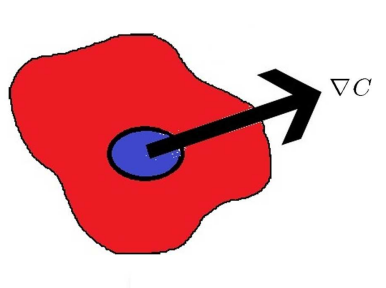

(b)
Figure 3. The procedure for calculating the concentration gradient: a) Cell concentration limits; and b) total gradient resulted from the superposition principle.

3. The spring connecting the nucleus to the leading edge is designated;

4. The stiffness of this spring is increased, causing a net force acting on the cell;

5. $\quad \vec{V}_{c}$, the velocity of the cell, resulting from forces acting upon it, due to external signals, is calculated.

In the mentioned cell simulator, the field of biochemical factor concentration has been solved using the computational fluid dynamics method, so the simulator would be able to check the position of the cell and calculate the concentration difference between the two ends of the cell in two independent directions, as shown in Figure 3.

Therefore, the direction of the gradient can be calculated by the superposition of the gradients in the two perpendicular directions (see Figure 3(b)).

Based on experimental observations, migrating cells tend to maintain their direction of motion, [17]. It means that the cells have a resistance against changing their velocities, so, this force is like inertia inside the cells to maintain its instantaneous velocity. In this simulation, when the velocity of the cell is refreshed at each time step, the new velocity is calculated, as the weighted mean of cell velocity from the previous time step. The $\vec{V}_{m}$ component appearing in Eq. (5) is the velocity of migration from the previous time step, $\vec{V}_{m}^{\text {past }}$.

The last factor contributing to cell migration is the random walks of the cell. Random motions are considered in the modeling of cell migration in order to include the effects of unknown factors. In this study, at each time step, two random numbers are generated which are used to build a random velocity component, $\vec{V}_{r} .5$

\section{Tuning the migration components coefficients}

After defining various components accounting for cell migration, there are a few constants associated with each component that should be determined before running the cell migration simulation. Translating the chemo attractant concentration gradient direction into a specified force, and identifying random constants, memory constants and drag force constants are important steps that should be determined to complete the cell migration simulator code. As no specific documented data is available in the literature, in this regard, we chose an alternative method in order to find the relevant constant parameters. For doing this, we used experimental movies of chemotacting cells. The experimental videos are regarding brain derived endothelial cells migrating for a period of 12 hours in response to concentration gradients of the forebrain embryonic cortical neuron Conditioned Medium (CM). This factor was previously shown to induce the directional chemotaxis of endothelial cells with over-expressed G protein coupled protein 124 (GPR 124) [18]. By comparing various cell movement parameters, like cell displacement and its mean velocity, with those of the cell simulator code, we calculated the mentioned constant parameters.

\subsection{Random walk constant}

In order to find the random walk constant, we analyzed the experimental video of chemotacting cells for 12 hours. Since the imposed concentration gradient is in a $Y$-direction, any movement in the $X$-direction is a result of random movements of the cell. The root mean square of positions of the cells after each one hour was calculated for a total time of 12 hours, and was plotted against the square root of time. As we know, the root mean square variation of the positions of a random walker is linear against the square root of time, which is evident in both the experimental data and simulation results in Figure 4. Afterwards, a straight line was fitted to the plot and its slope was found. Using a trial and error approach, we ran the simulation for different values of random walk constant, and chose the value in which the slope of $x_{r m s}$ plot versus square root of time for the simulation matched that of experimental data. As a result, we came up with a random walk constant in which the cell random migration of simulation was consistent with that of experimental videos (see Figure 4).

\subsection{Chemotaxis tuning}

After setting up the constant of the random walk in the simulation, the relevant parameter of the chemotaxis was identified. As mentioned previously, the chemotaxis is simulated by identifying the leading edge nodes and then increasing the relevant spring stiffness by a constant chemotaxis factor. In order to find a reasonable value for this factor, we repeated a trial and error approach similar to that done for the random walk.

Since, in the experimental setup, the concentra- 


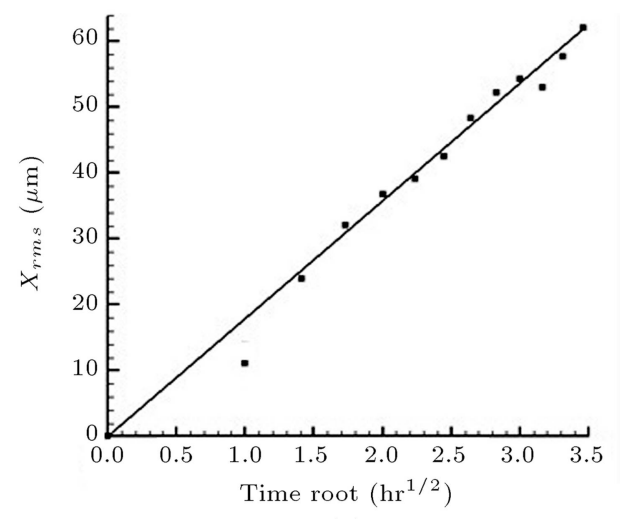

(a)

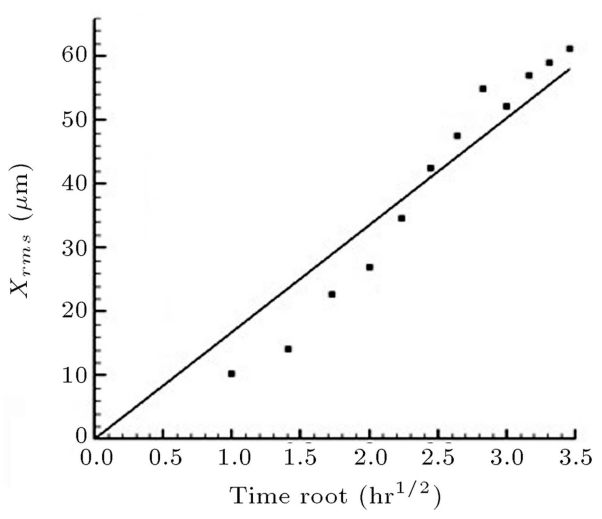

(b)

Figure 4. Comparison of random migration displacement, $x_{r m s}$ versus square root of time: (a) Experimental data (slope of the fitted line $=17.96 \frac{\mu \mathrm{m}}{\mathrm{Hr}}^{0.5}$ ); and $(\mathrm{b})$ simulation data (slope of the fitted line $=17.52 \frac{\mu \mathrm{m}}{\mathrm{Hr}}^{0.5}$ ).

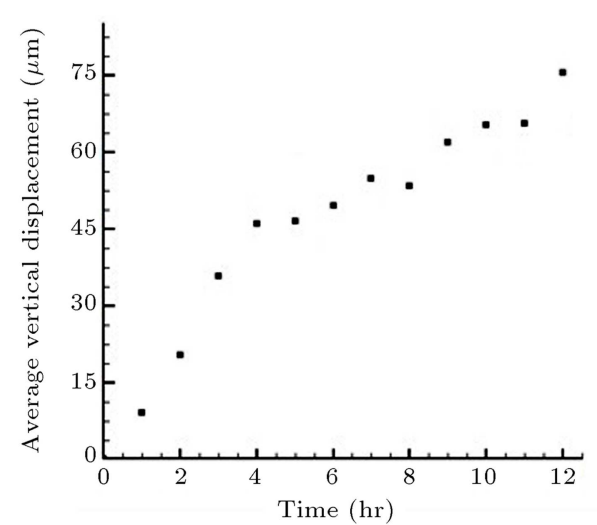

(a)

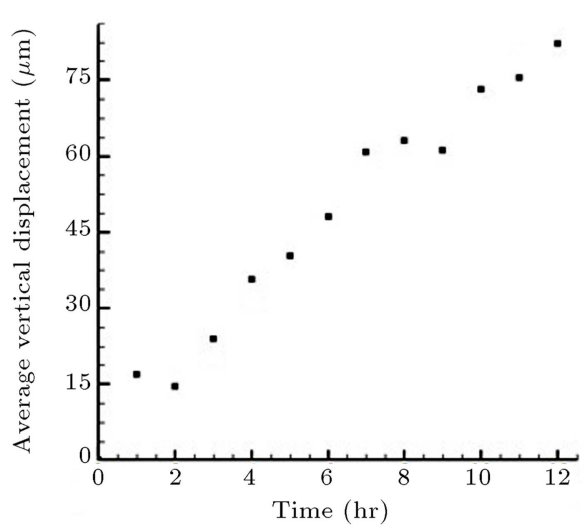

(b)

Figure 5. Comparison of average vertical displacement of chemotacting cell versus time in order to tune the chemotaxis effect coefficient: (a) Experimental data; and (b) simulation data.

tion gradient is in the $Y$-direction, it makes sense to find the chemotaxis constant by comparing the displacement of migrating cells in the movie and simulation in the $Y$-direction. Since the random walk constant has already been calculated and referred to the code, there is no concern regarding the effects of random walk in the chemotaxis of the cells in the $Y$ direction. The average vertical displacement of the migrating cells versus time is shown in Figure 5 for experimental data and simulation results. Again, we found the chemotaxis constant using a trial and error approach, so that the simulation curves converge to experimental ones.

It could be concluded from Figures 4 and 5 that the cell migrating simulator has an acceptable agreement with experimental results. As the chemotaxis phenomenon is semi-stochastic, a better agreement can be obtained by further simulations and experiment comparisons.

\subsection{Drag force coefficient}

The final step in tuning the cell simulator code is finding the viscous drag coefficient. In choosing this constant, we turned our attention to the mean velocity of migrating cells instead of their displacement. The drag force was set to be proportional to the maximum of random walk force by an unknown factor. The unknown coefficient was found in such a way that limiting the velocity of migrating cells in the simulation converges to the average velocity of the migrating cells in the experimental video.

At this step, all the necessary parameters and coefficients of the cell migration model are identified and fixed. By obtaining the velocity of the cells, their motions are simply demonstrated by applying the velocity-Verlet method.

The obtained constants are displayed in Table 1.

Table 1. Numerical values of parameters calibrated in the cell simulator.

\begin{tabular}{lc}
\hline Parameters & $\begin{array}{c}\text { Order of magnitude of } \\
\text { the constant value }\end{array}$ \\
\hline Random force & $\mathrm{e}-14(\mathrm{~N})$ \\
Drag force & $\frac{10^{-14} \mathrm{~N}}{50 \frac{\mu m}{h}}$ \\
Internal forces & According to $[15]$ \\
\hline
\end{tabular}


It should be considered that these constants are strongly correlated with the type of cell, as well as the type of biochemical factor used. Indeed, in order to find the calibrated constants in the cell simulator for other cells with different types of biochemical factor, specific related experiments are needed.

\section{Migration results and discussion}

In this study, a cell simulator is developed to model the cell responses to the imposed chemical gradients in the extracellular medium. The positioning of the nodes related to the simulated migrating cell have been shown in Figure 6. It can be seen in this figure that the cell has a tendency to migrate in the gradient direction, but the random walk is able to change the direction of motion.

Figure 7 displays the migrating cellmorphology

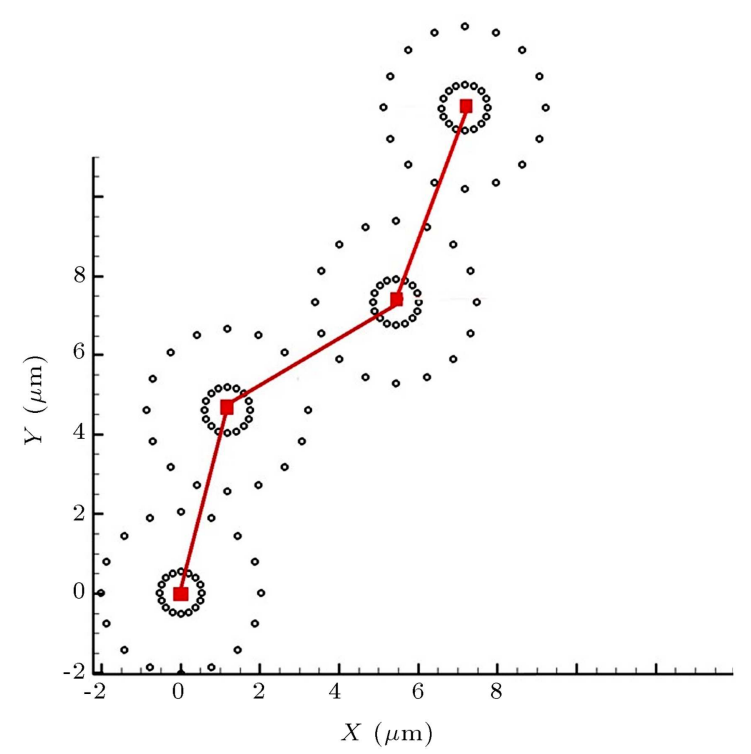

Figure 6. First few steps of a single cell migration. Membrane and nucleus nodes are shown in this figure.

The cell tends to migrate in a vertical direction as a result of imposed gradient, while the random walk effects deviates the cell direction from pure vertical movement.

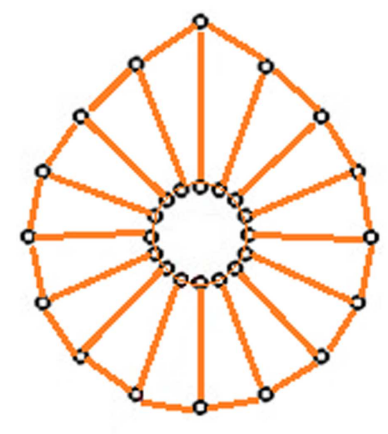

(a)

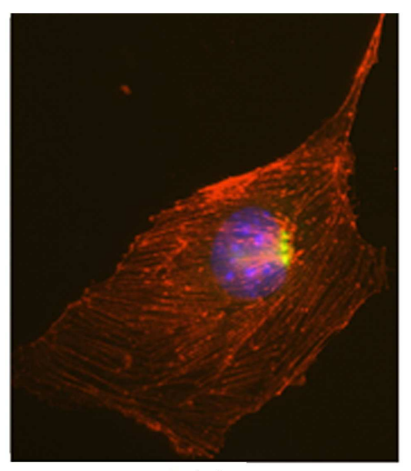

(b)
Figure 7. The morphology of a migrating cell obtained from cell simulation.

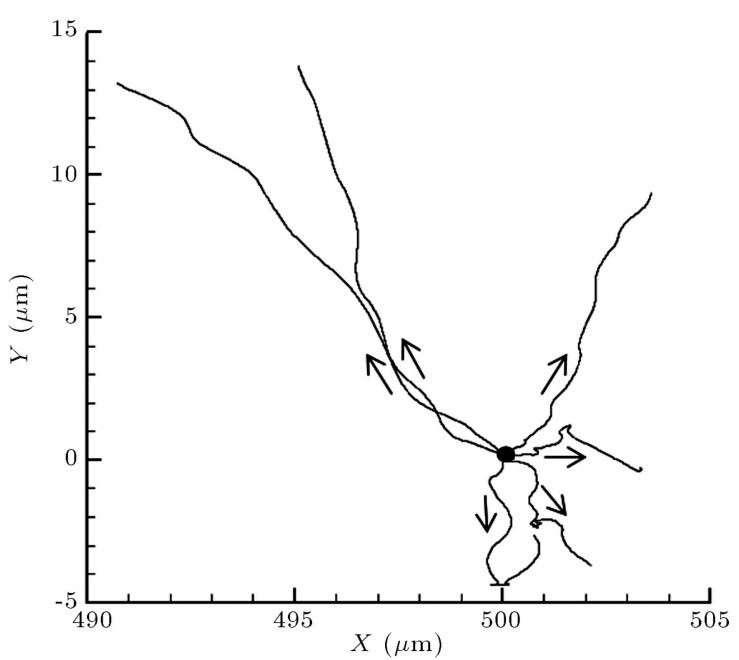

Figure 8. Trajectories of migrating cells, all initially at the same location. The cell's tendency to migrate in the direction of the imposed concentration gradient is evident.

obtained from simulator results, in which the reorganization of the cytoskeleton is observable. Indeed, this figure makes a qualitative demonstration of the formation of the lamellipodia due to cytoskeleton reorganization. In Figure 8, the trajectories of the cells that were initially positioned in the same place are illustrated. As can be seen in this figure, the overall motion of the cells lies in the direction of the gradient, but when exposed to a constant gradient, the cell would not migrate through a unique path. This is the result of the random walk navigations of the cells. The cell trajectories in Figure 8 are not the same because of the stochastic velocity component, $\vec{V}_{r}$. However, all trajectories are, generally, in the direction of the gradient when observed for a considerable time.

\section{Conclusion}

In this project, cell motility during chemotaxis was studied by computational methods. The Voigt model was applied and a cell model, including nucleus, cytoskeleton, and membrane, was developed. The migration of this cell model through the extracellular gradient was attributed to four factors discussed.

The presented method could be used in the study of the mechanisms of cardiovascular diseases and their potential treatment, such as cancer treatment. Thus, studying the mechanism of anti-angiogenic and pro-angiogenic therapies is one of the basic aims of this work. Also, the cell migration simulator code developed in this study, can be extended to study the migration of similar types of cell with other varying biochemical factors, or by varying concentration profiles by updating various cell migration parameters introduced in this study. The method proposed in this study can make it unnecessary to undertake expensive 
experiments to investigate the response of cells in the presence of various biochemical concentration gradients.

As shown in Figures 6 and 8, the method presented in this work has the capability of offering distinct software for cell migration. Thus, one aspect of future work to complete this simulation is to develop a software pack in cell migration. Implementing the effects of cell-cell interactions in the simulator, in order to study group migration of cells, is further possible work in this field. Enhancing cell cytoskeletal detail is another modification that improves the precision of the simulation that is to be performed.

\section{Conflict of interest}

There is no conflict of interest regarding this study.

\section{References}

1. Lauffenburger, D.A. and Horwitz, A.F. "Cell migration: A physically integrated molecular process", Cell, 84(3), pp. 359-369 (1996).

2. Ridley, A.J., Schwartz, M.A., Burridge, K., Firtel, R.A., Ginsberg, M.H., Borisy, G., Parsons, J.T. and Horwitz, A.R. "Cell migration: Integrating signals from front to back", Science, 302(5651), pp. 1704-1709 (2003).

3. Servant, G., Weiner, O.D., Herzmark, P., Balla, T., Sedat, J.W. and Bourne, H.R. "Polarization of chemoattractant receptor signaling during neutrophil chemotaxis", Science, 287(5455), pp. 1037-1040 (2000).

4. Franz, C.M., Jones, G.E. and Ridley, A.J. "Cell migration in development and disease", Developmental Cell, 2(2), pp. 153-158 (2002).

5. Meinhardt, H. "Orientation of chemotactic cells and growth cones: models and mechanisms", Journal of Cell Science, 112(17), pp. 2867-2874 (1999).

6. Narang, A., Subramanian, K. and Lauffenburger, D.A. "A mathematical model for chemoattractant gradient sensing based on receptor-regulated membrane phospholipid signaling dynamics", Annals of Biomedical Engineering, 29(8), pp. 677-691 (2001).

7. Ma, L., Janetopoulos, C., Yang, L., Devreotes, P.N. and Iglesias, P.A. "Two complementary, local excitation, global inhibition mechanisms acting in parallel can explain the chemoattractant-induced regulation of PI $(3,4,5)$ P3 response in dictyostelium cells", Biophysical Journal, 87(6), pp. 3764-3774 (2004).

8. Levine, H., Kessler, D.A. and Rappel, W.J. "Directional sensing in eukaryotic chemotaxis: A balanced inactivation model", Proceedings of the National Academy of Sciences, 103(26), pp. 9761-9766 (2006).

9. Swaney, K.F., Huang, C.H. and Devreotes, P.N. "Eukaryotic chemotaxis: A network of signaling pathways controls motility, directional sensing, and polarity", Annual Review of Biophysics, 39, pp. 265-289 (2010).

10. Neilson, M.P., Veltman, D.M., van Haastert, P.J., Webb, S.D., Mackenzie, J.A. and Insall, R.H. "Chemotaxis: A feedback-based computational model robustly predicts multiple aspects of real cell behaviour", PLoS Biology, 9(5), e1000618 (2011).

11. Andrew, N. and Insall, R.H. "Chemotaxis in shallow gradients is mediated independently of PtdIns 3-kinase by biased choices between random protrusions", $\mathrm{Na}$ ture Cell Biology, 9(2), pp. 193-200 (2007).

12. Killich, T., Plath, P.J., Haß, E.C., Xiang, W., Bultmann, H., Rensing, L. and Vicker, M.G. "Cell movement and shape are non-random and determined by intracellular, oscillatory rotating waves in Dictyostelium amoebae", Biosystems, 33(2), pp. 75-87 (1994).

13. Killich, T., Plath, P.J., Wei, X., Bultmann, H., Rensing, L. and Vicker, M.G. "The locomotion, shape and pseudopodial dynamics of unstimulated Dictyostelium cells are not random", Journal of Cell Science, 106(4), pp. 1005-1013 (1993).

14. Kiyoumarsioskouei, A., Saidi, M.S. and Firoozabadi, B. "An endothelial cell model containing cytoskeletal components: Suspension and adherent states", Journal of Biomedical Science \& Engineering, 5(12), pp. 737742 (2012).

15. Jamali, Y., Azimi, M. and Mofrad, M.R. "A subcellular viscoelastic model for cell population mechanics", PloS One, 5(8), e12097 (2010).

16. Whitman, M., Downes, C.P., Keeler, M., Keller, T. and Cantley, L. "Type I phosphatidylinositol kinase makes a novel inositol phospholipid, phosphatidylinositol-3-phosphate", Nature, 332(6165), pp. 644-646 (1988).

17. Zaman, M.H., Kamm, R.D., Matsudaira, P. and Lauffenburger, D.A. "Computational model for cell migration in three-dimensional matrices", Biophysical Journal, 89(2), pp. 1389-1397 (2005).

18. Shamloo, A. "Cell-cell interactions mediate cytoskeleton organization and collective endothelial cell chemotaxis", Cytoskeleton, 71(9), pp. 501-512 (2014).

\section{Biographies}

Amir Kiyoumarsioskouei was born in Osku, Iran. He obtained his BS degree in Mechanical Engineering from Amir Kabir University of Technology, in 2009, and is currently a PhD degree candidate in Sharif University of Technology, Tehran, Iran.

Amir Shamloo received his $\mathrm{PhD}$ degree from Stanford University, Stanford, CA, USA, in 2010, and became a postdoctoral scholar at the University of California, Berkeley, CA, USA. He is currently Assistant Professor in the Mechanical Engineering Department of Sharif University of Technology, Tehran, Iran. His 
research involves the design and fabrication of microfluidic devices and their application in biology, and cell and tissue mechanics.

Sajjad Azimi obtained his BS degree in Mechanical Engineering, in 2012, from Sharif University of Technology, Tehran, Iran, where he is currently an MS degree student. His field of research study is the modeling of biological systems and microfabrication.

Mohammad Abeddust received his BS degree in Mechanical Engineering from Shiraz University, in 2012, followed by an MS degree in the same subject from Sharif University of Technology, Tehran, Iran, in 2015.
His research interests include numerical investigation of thermo-fluidic systems, with particular interest in biological systems. $\mathrm{He}$ is also familiar with optical measurement techniques in fluid mechanics and heat transfers.

Mohammad Said Saidi received his $\mathrm{PhD}$ degree from Massachusetts Institute of Technology, USA, in 1979, and is currently Professor of Mechanical Engineering at Sharif University of Technology, Tehran, Iran. His research interests include modeling and numerical analysis of the transport and deposition of aerosol particles, biofluids, thermal-hydraulics of porous media, and microchannels 